UDC 621.396.67

\title{
DOMINANT T-MODE OF A CRUCIFORM WAVEGUIDE
}

\author{
S. E. Bankov
}

Kotelnikov Institute of Radioengineering and Electronics of Russian Academy of Sciences, Mokhovaya 11-7, Moscow 125009, Russia

The paper is received on July 26, 2019

\begin{abstract}
The problem of T-mode propagation in the cruciform waveguide is solved. The boundary problem is reduced to an integral equation that is solved with help of Galerkin's method. Expressions for dominant eigen mode field components are obtained. They are used for characteristic impedance calculation. Results of numerical simulation of characteristic impedance behavior are presented. They are compared with results obtained by HFSS, and good agreement between them is demonstrated.
\end{abstract}

Key words. T-mode, cruciform waveguide, Galerkin's method, characteristic impedance.

\section{Problem formulation}

Development of an ultra-wideband (UWB) antenna arrays is an actual problem that was considered in many works (see for example [1], [2]). Factors that prevent UWB antenna array operating frequency range (OFR) expansion are noted in several papers [3], [4]. One of them is a transition connecting different transmission lines (TRL). Such device has a limited OFR that determines array OFR in total. For example widely known in UWB applications Vivaldi antenna contains transition from microstrip to slot line [5]. Usually the transition connects TRL with a standard value of characteristic impedance close to $50 \mathrm{Ohm}$. In these conditions it is very difficult to obtain OFR greater than 10:1 (we define OFR as a relation of maximum frequency $F_{\max }$ to minimum frequency $F_{\min }$ ). Attempts to construct UWB array without above transition presented in [6] and [7] were not successful. Considered in these works arrays do not operate in a proper way because of parasitic effects of anomalous backward radiation [6] and impedance anomalies [7]. 
New concept of UWB array - so called dual section array was presented in [8]. This array also contains transition connecting different TRL. However these TRL have relatively low impedance in the point of connection. As it was demonstrated in [8] application of low impedance TRL increases array OFR thus that noted above limit 10:1 may be overcame.

In frame of dual section array concept application of slot lines for electromagnetic wave radiation is not convenient. Naturally slot line has relatively high impedance and extremely narrow slots are required for lines with impedance sufficiently lower than $50 \mathrm{Ohm}$. Thus another TRL with naturally low impedance should be used in dual section arrays. Planar waveguide was proposed in [8] as such TRL for single polarization array.

More complicated transmission media is required for dual polarization array. It should support propagation of two modes with different polarizations. A 2D array of metal rods with square cross section may be used as such transmission media (see fig. 1). Eigen modes of this structure have extremely small impedance when distance between rods is enough small and their impedance is close to free space impedance when rod size is close to zero. Thus this structure with variable rod size is good for application as a second section of dual section array.

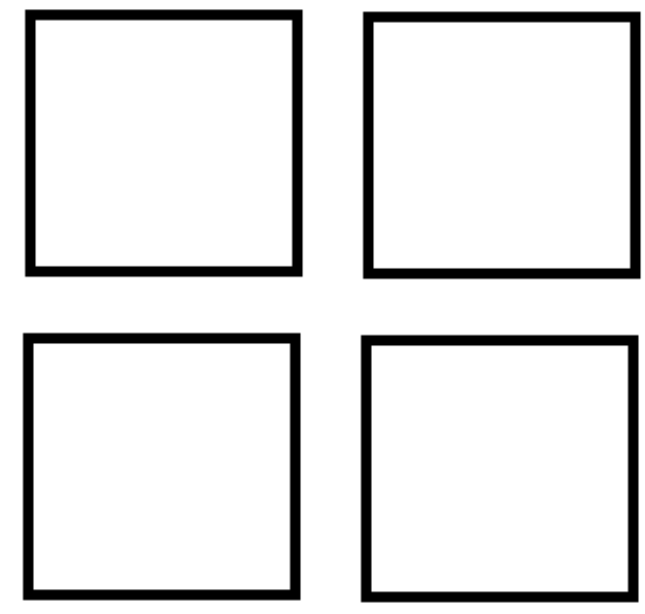

Fig. 1. 2D array of square metal rods

We may also consider array of metal rods as an impedance transformer. Dependence of array eigen mode impedance on geometrical parameters is required for correct transformer design. One has to solve an eigen mode boundary problem to 
obtain this dependence. Solution of the problem is the main subject of this work.

Array eigen modes depend not only geometrical parameters but they also depend on scan angles or phase shifts between array periods. In this work we consider particular case of in-phase infinite 2D array of square metal rods. It is a known fact that infinite array analysis in quasi periodical regime may be reduced to analysis of one array period (see fig. 2) - so called Floquet channel [9]. Floquet channel is a waveguide of special type with periodical boundary conditions on its walls.

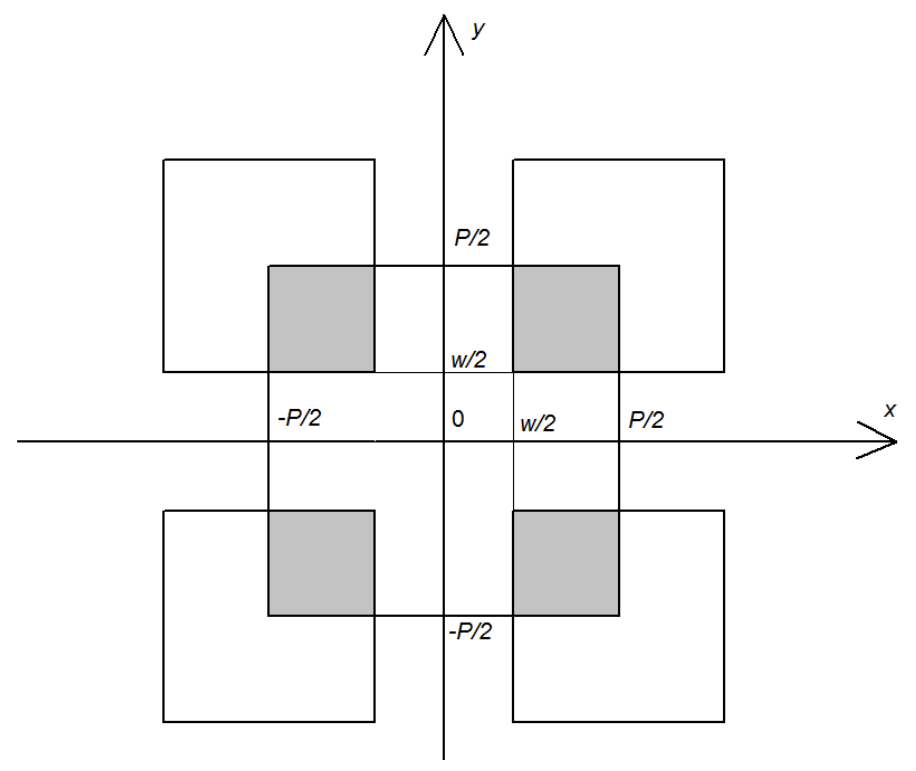

Fig. 2. Array period.

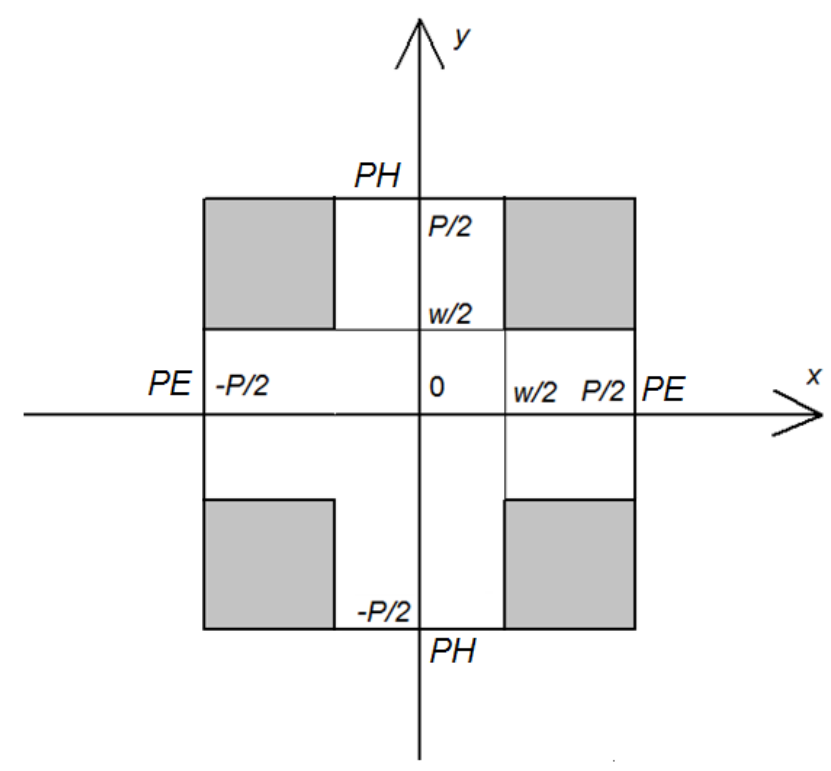

Fig. 3. Cruciform waveguide. 
For in-phase array walls with periodical boundary conditions may be replaced by more simple perfect electric (PE) and magnetic (PH) walls if array period has planes of symmetry as it is shown in fig. 3 . Thus we obtain cruciform waveguide that is the main subject of our work.

\section{Boundary problem formulation and solution}

Waveguide cross section (fig. 3) has planes of symmetry $X O Z$ and $X O Y$. Therefore there are eigen modes with equal to zero at $x, y= \pm P / 2$ tangential components of electric and magnetic fields. Due to it we may place in the corresponding planes perfect electric or perfect magnetic walls and analyze one quarter of the waveguide.

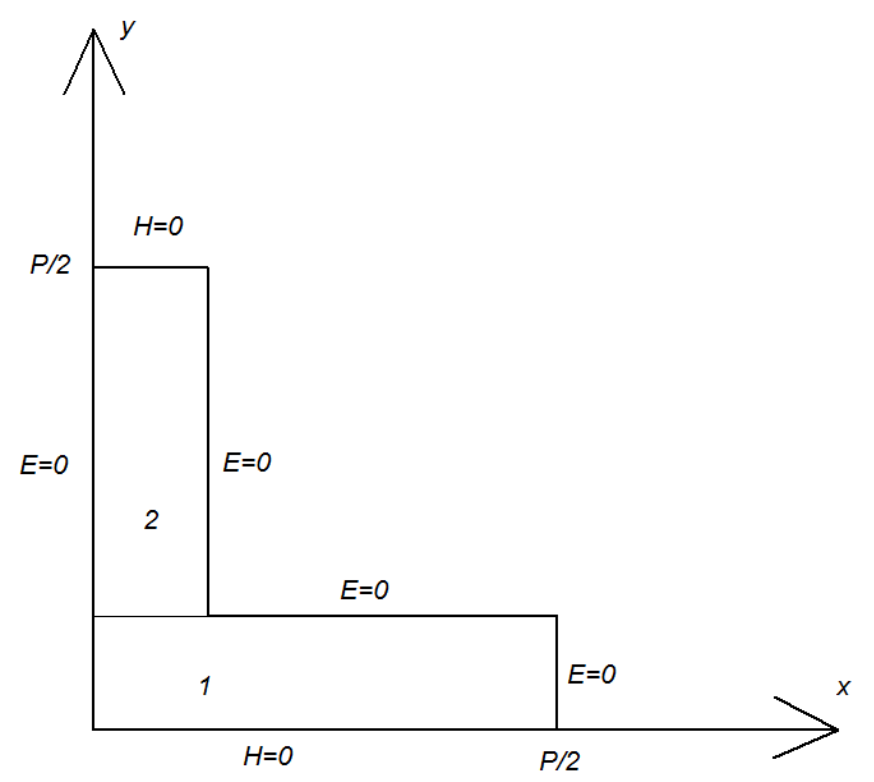

Fig. 4. Quarter of cruciform waveguide.

The most interesting for our analysis combination of walls is shown in fig. 4 . One may see that the structure contains two isolated conductors and therefore the dominant mode of the waveguide is a transversal $T$-mode. Next let us divide inner part of waveguide cross section onto two regions 1,2 as it is shown in fig. 4 . We present fields inside regions 1,2 in form of the following expansions:

$$
E_{z 1}=\left(k^{2}-\beta^{2}\right) \sum_{n=0}^{\infty} A_{n} \sin \kappa_{1 n} x \cosh \gamma_{1 n} y,
$$




$$
\begin{aligned}
& E_{z 2}=\left(k^{2}-\beta^{2}\right) \sum_{n=0}^{\infty} B_{n} \sin \kappa_{2 n} x \cosh \gamma_{2 n}(P / 2-y), \\
& \kappa_{1 n}=\frac{2 \pi n}{P} \\
& \kappa_{2 n}=\frac{2 \pi n}{w} \\
& \gamma_{1 n}=\sqrt{\kappa_{1 n}^{2}+\beta^{2}-k^{2}} \\
& \gamma_{2 n}=\sqrt{\kappa_{2 n}^{2}+\beta^{2}-k^{2}}
\end{aligned}
$$

here $\beta$ is a propagation constant, $A_{n}, B_{n}$ - unknown coefficients, $k$ - free space wavenumber. In formulas (1) common for all expressions term $\exp (-i \beta z)$ is omitted. Presence of multipliers $\left(k^{2}-\beta^{2}\right)$ will be explained below.

Next let us find field components $E_{x}, H_{x}$, with help of known relations [10]:

$$
\begin{aligned}
& E_{x 1}=-i \beta \sum_{n=0}^{\infty} A_{n} \kappa_{1 n} \cos \kappa_{1 n} x \cosh \gamma_{1 n} y, \\
& E_{x 2}=-i \beta \sum_{n=0}^{\infty} B_{n} \kappa_{2 n} \cos \kappa_{2 n} x \cosh \gamma_{2 n}(P / 2-y), \\
& H_{x 1}=\frac{i k}{W_{0}} \sum_{n=0}^{\infty} A_{n} \gamma_{1 n} \sin \kappa_{1 n} x \sinh \gamma_{1 n} y, \\
& E_{z 2}=\frac{i k}{W_{0}} \sum_{n=0}^{\infty} B_{n} \gamma_{2 n} \sin \kappa_{2 n} x \sinh \gamma_{2 n}(P / 2-y),
\end{aligned}
$$

where $W_{0}$ - free space wave impedance.

Now we may express coefficients $A_{n}, B_{n}$ through electric field $\mathrm{E}(x)$ in plane $y=w / 2$ : 


$$
\begin{aligned}
& A_{n}=-\frac{2 \varepsilon_{n}}{i \beta \kappa_{1 n} P \operatorname{ch} \gamma_{1 n} w / 2} \int_{0}^{w / 2} \mathrm{E}(x) \cos \kappa_{1 n} x d x, \\
& B_{n}=-\frac{2 \varepsilon_{n}}{i \beta \kappa_{2 n} w \operatorname{ch} \gamma_{2 n} a / 2} \int_{0}^{w / 2} \mathrm{E}(x) \cos \kappa_{2 n} x d x, \\
& \varepsilon_{n}=\left\{\begin{array}{l}
1, n=0, \\
2, n>0,
\end{array}\right. \\
& a=P-w .
\end{aligned}
$$

Let us note that T-wave propagation is equal to $k$. It is easy to see that in this case longitudinal field components are equal to zero while transversal components have finite values. Such field behavior explains presence in expressions (1) terms $\left(k^{2}-\beta^{2}\right)$.

The following expressions are also valid for $T$-wave:

$$
\gamma_{1,2 n}=\kappa_{1,2 n} .
$$

Writing coefficients in form (4) we automatically satisfy boundary conditions at $y=w / 2$. Thus next we have to satisfy boundary conditions of magnetic field continuity at $y=w / 2,0<x<w / 2$. Substituting relations (4) to expressions for magnetic field in regions 1,2 and equating them we obtain required integral equation for electric $\mathrm{E}(x)$ :

$$
\begin{aligned}
& 4 \int_{0}^{w / 2} \mathrm{E}\left(x^{\prime}\right) \sum_{n=1}^{\infty}\left(\frac{\operatorname{th} \kappa_{1 n} w / 2}{P} \sin \kappa_{1 n} x \cos \kappa_{1 n} x^{\prime}+\right. \\
& \left.\frac{\operatorname{th} \kappa_{2 n} a / 2}{w} \sin \kappa_{2 n} x \cos \kappa_{2 n} x^{\prime}\right) d x^{\prime}=0, \\
& 0<x<w / 2 .
\end{aligned}
$$

Galerkin's method is a typical way for integral equations solution. However it is not convenient to apply it directly to equation (6) because it has non symmetrical kernel. Let us integrate expression (6) over variable $x$ to obtain new form of integral equation with symmetrical kernel: 


$$
\begin{aligned}
& 4 \int_{0}^{w / 2} \mathrm{E}\left(x^{\prime}\right) \sum_{n=1}^{\infty}\left(\frac{\operatorname{th} \kappa_{1 n} w / 2}{\kappa_{1 n} P} \cos \kappa_{1 n} x \cos \kappa_{1 n} x^{\prime}+\right. \\
& \left.\frac{\text { th } \kappa_{2 n} a / 2}{\kappa_{2 n} w} \cos \kappa_{2 n} x \cos \kappa_{2 n} x^{\prime}\right) d x^{\prime}=C,
\end{aligned}
$$

where $C$ a constant of integration.

We may apply Galerkin's method to integral equation (7) solution because now left part of expression (7) identically depends on arguments $x, x^{\prime}$. In accordance with Galerkin's method we present unknown function as the following expansion:

$$
\mathrm{E}(x)=\sum_{m=0}^{\infty} R_{m} f_{m}(x)
$$

where $f_{m}(x)$ are known base functions and $R_{m}$ are unknown coefficients.

Let us substitute expression (8) in equation (7) and find projection of the equation on the same system of test functions (8). In frame of Galerkin's method test functions coincide with base functions (8). As a result we obtain system of linear algebraic equation (SLAE) relatively coefficients $R_{m}$ :

$$
\begin{aligned}
& \sum_{q=0}^{\infty} Z_{p, q} R_{q}=S_{p}, \\
& Z_{p, q}=4 \sum_{n=1}^{\infty}\left(\frac{\operatorname{th} \kappa_{1 n} w / 2}{\kappa_{1 n} P} I_{1 n, p} I_{1 n, q}+\frac{\mathrm{th} \kappa_{2 n} a / 2}{\kappa_{2 n} w} I_{2 n, p} I_{2 n, q}\right), \\
& S_{p}=C I_{10, p}, \\
& I_{1 n, p}=\int_{0}^{w / 2} \cos \kappa_{1 n} x f_{p}(x) d x, \\
& I_{2 n, p}=\int_{0}^{w / 2} \cos \kappa_{2 n} x f_{p}(x) d x .
\end{aligned}
$$

Choice of base functions is an important step of Galerkin's method. These functions should maximally takes into account information about field behavior. In our case such information is field behavior near the edge of perfectly conducting 
wedge with $90^{\circ}$ angle that is located in point $x=w / 2$. Meixner conditions determine field behavior in wedge type regions [11]. The following system of base functions takes them into account:

$$
f_{m}(x)=\frac{\cos \kappa_{2 m} x}{\left(\left(\frac{w}{2}\right)^{2}-x^{2}\right)^{1 / 3}}
$$

Integrals $I_{1,2, n, p}$ are expressed through Bessel functions [12]:

$$
\begin{aligned}
& I_{1,2, n, m}=\frac{\sqrt{\pi}}{4}\left(\left(\frac{w}{\kappa_{1,2 n}+\kappa_{2 m}}\right)^{1 / 6} J_{1 / 6}\left(\left(\kappa_{1,2 n}+\kappa_{2 m}\right) w / 2\right)+\right. \\
& \left.\left(\frac{w}{\kappa_{1,2 n}-\kappa_{2 m}}\right)^{1 / 6} J_{1 / 6}\left(\left(\kappa_{1,2 n}-\kappa_{2 m}\right) w / 2\right)\right) \Gamma(2 / 3),
\end{aligned}
$$

where $J_{1 / 6}$ is Bessel function with order $1 / 6, \Gamma$ is Gamma function.

SLAE (9) solution determines $R_{m}$ proportional to arbitrary constant $C$ that is typical for eigen mode problems. This constant may be unambiguously find only as a solution of waveguide excitation problem.

\section{3. $T$-wave characteristic impedance}

After SLAE (9) solution we may find eigen mode field and characteristic impedance $Z_{c}$. We define it as a relation of voltage $U$ to current $J$ :

$$
\begin{aligned}
& Z_{c}=U / J, \\
& U=\int_{0}^{w / 2} \mathrm{E}_{x}(x) d x, J=\left|\int_{0}^{P / 2} H_{y}(0, y) d y\right| .
\end{aligned}
$$

Coefficients $A_{n}, B_{n}$ should be expressed through coefficients $R_{m}$ that are found from SLAE (9) solution: 


$$
\begin{aligned}
& A_{n}=-\frac{2 \varepsilon_{n}}{i k \kappa_{1 n} P \operatorname{ch} \kappa_{1 n} w / 2} \sum_{m=0}^{\infty} R_{m} I_{1 n, m}, \\
& B_{n}=-\frac{2 \varepsilon_{n}}{i k \kappa_{2 n} w \operatorname{ch} \kappa_{2 n} a / 2} \sum_{m=0}^{\infty} R_{m} I_{2 n, m} .
\end{aligned}
$$

$T$-wave components $H_{y}$ and $E_{x}$ are connected by the simple relation:

$$
H_{y}=E_{x} / W_{0} .
$$

With help of the last notes we find:

$$
\begin{aligned}
& U=\sum_{m=0}^{\infty} R_{m} I_{20, m}, \\
& J=\frac{1}{W_{0}} \sum_{n=0}^{\infty} 2 \varepsilon_{n} \sum_{m=0}^{\infty} R_{m}\left(\frac{I_{1 n, m} \mathrm{th} \kappa_{1 n} w / 2}{\kappa_{1 n} P}+\frac{I_{2 n, m} \mathrm{th} \kappa_{2 n} a / 2}{\kappa_{2 n} w}\right), \\
& Z_{c}=\frac{W_{0} \sum_{m=0}^{\infty} R_{m} I_{20, m}}{\sum_{n=0}^{\infty} 2 \varepsilon_{n} \sum_{m=0}^{\infty} R_{m}\left(\frac{I_{1 n, m} \mathrm{th} \kappa_{1 n} w / 2}{\kappa_{1 n} P}+\frac{I_{2 n, m} \mathrm{th} \kappa_{2 n} a / 2}{\kappa_{2 n} w}\right)}
\end{aligned}
$$

\section{Numerical algorithm convergence}

Let us next consider results of boundary problem numerical solution. Convergence of the described above algorithm is an important problem. Source of the solution error is a finite number of base functions $N$ and corresponding substitution of infinite series by finite sums. Parameter $N$ is usually selected from numerical experiments with help of criteria of solution independence on parameter $N$.

It is reasonable to introduce two groups of parameters: primary and secondary. Eigen mode field is our primary parameter. Eigen mode characteristic impedance is the secondary parameter that may be obtained through eigen mode field integration. Thus we may also consider characteristic impedance as an integral parameter.

Let us now consider convergence of primary parameter - eigen mode field. For 
it we should study dependence of electric field $E_{x}(x)$ on parameter $N$. Convergence of integral equation solution may be also described with help of parameter $\delta F$ that is defined by as follows:

$$
\delta F=\frac{2}{w} \int_{0}^{w / 2}\left|\frac{F(x)-C}{C}\right| d x,
$$

where $F(x)$ is the left part of equation (7). Parameter $\delta F$ characterizes tolerance with which boundary condition for magnetic field is satisfied.

Dependence of normalized electric field $E_{x}(x)$ on coordinate $x$ is shown in fig. 5 a,b. We normalize electric field on its value at $x=0$. Curves $1-4$ in fig. $5 \mathrm{a}$ are obtained for $P=15, N=2,4,6,8, w=1.7$, and in fig. $5 \mathrm{~b}$ for $w=11.4$. One may see a trend to solution of integral equation stabilization when $N$ is increased. Speed of convergence for electric field weakly depends on distance between $w$. We may state that for $N>8$ relative changes of electric field are negligibly small.

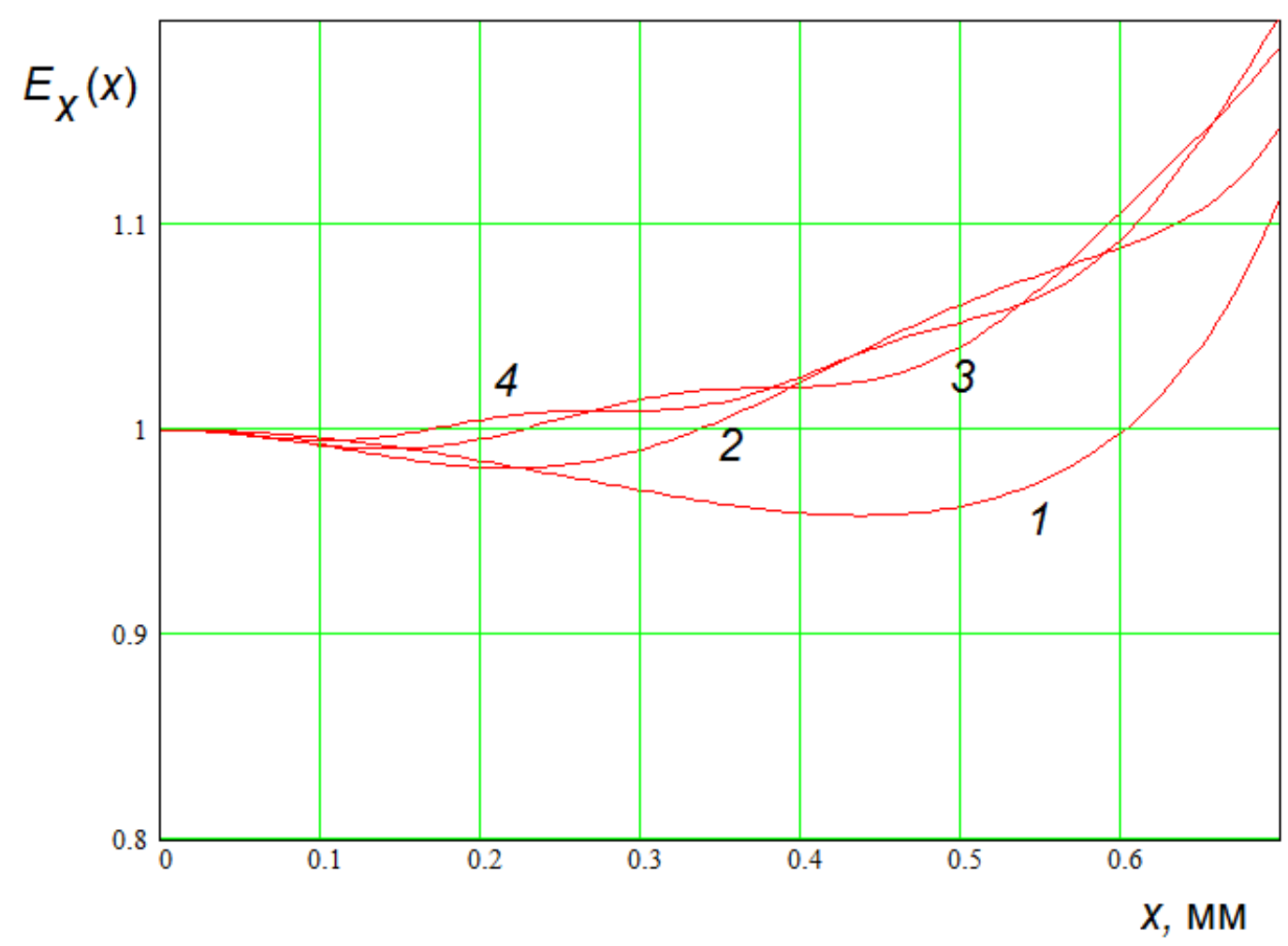




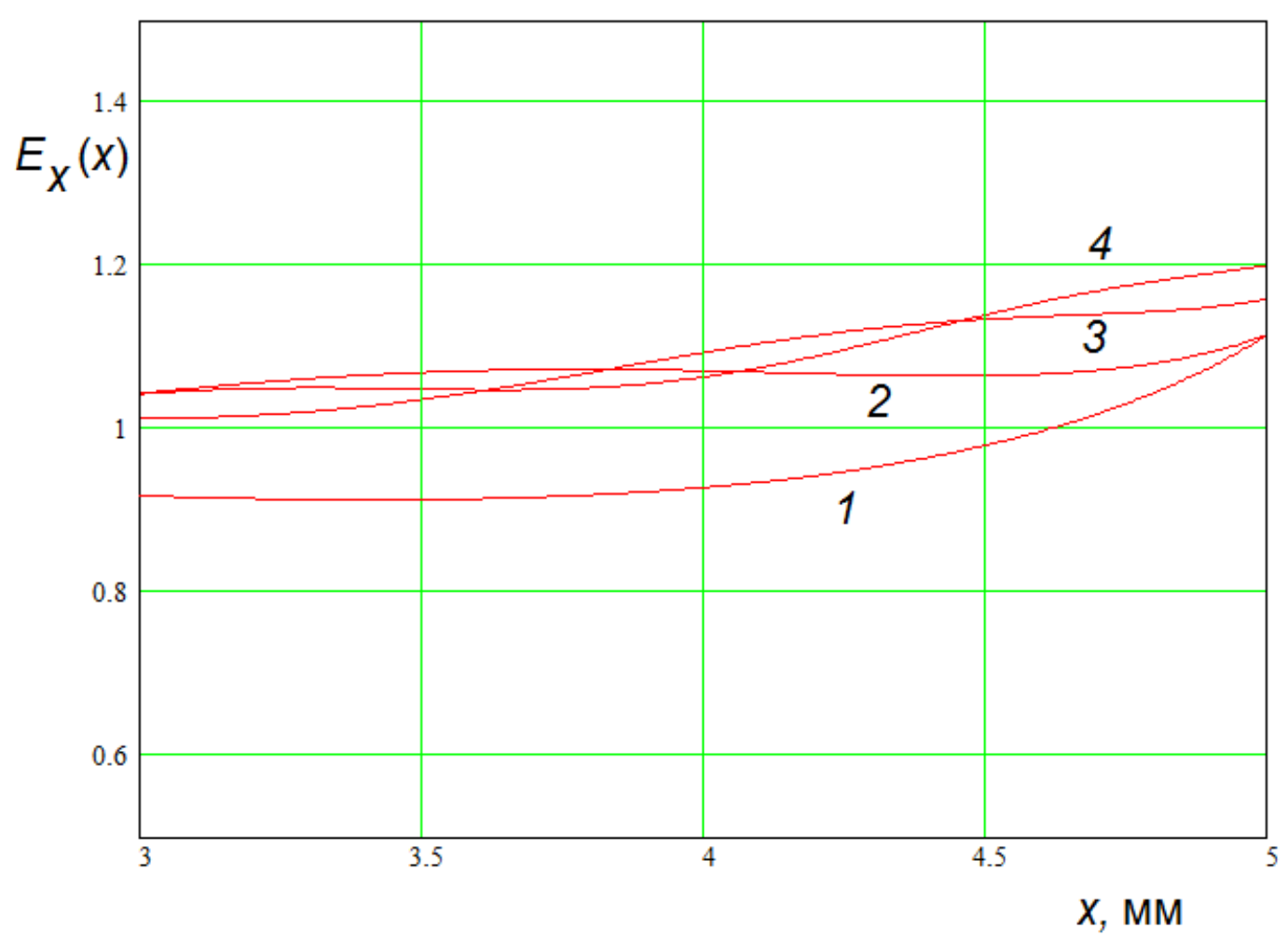

b

Fig. 5. Dependence of normalized electric field on coordinate $x$

Dependence of functional $\delta F$ on distance $w$ is presented in fig. 6. Curves 1 - 5 are calculated for $P=15, N=2,4,6,8,10$. It is seen that this parameter sufficiently depends on distance $w$. It rapidly grows with growth of $w$. Thus for big distances $w$ we have to apply relatively big number of base functions to obtain enough small value of parameter $\delta F$ than for small distances.

Convergence of integral parameter $Z_{c}$ sufficiently differs from primary parameters convergence. Dependence of characteristic impedance on parameter $N$ is presented in fig. 7 a,b. The curve in fig. 7 a is calculated for $P=15, w=1.7$ and in fig. $7 \mathrm{~b}$ for $w=11.4$. It is seen that the analyzed parameter weakly depends on number of base functions. It is also seen that speed of convergence weakly depends on distance $w$. Characteristic impedance is practically stable when $N \geq 4$. 


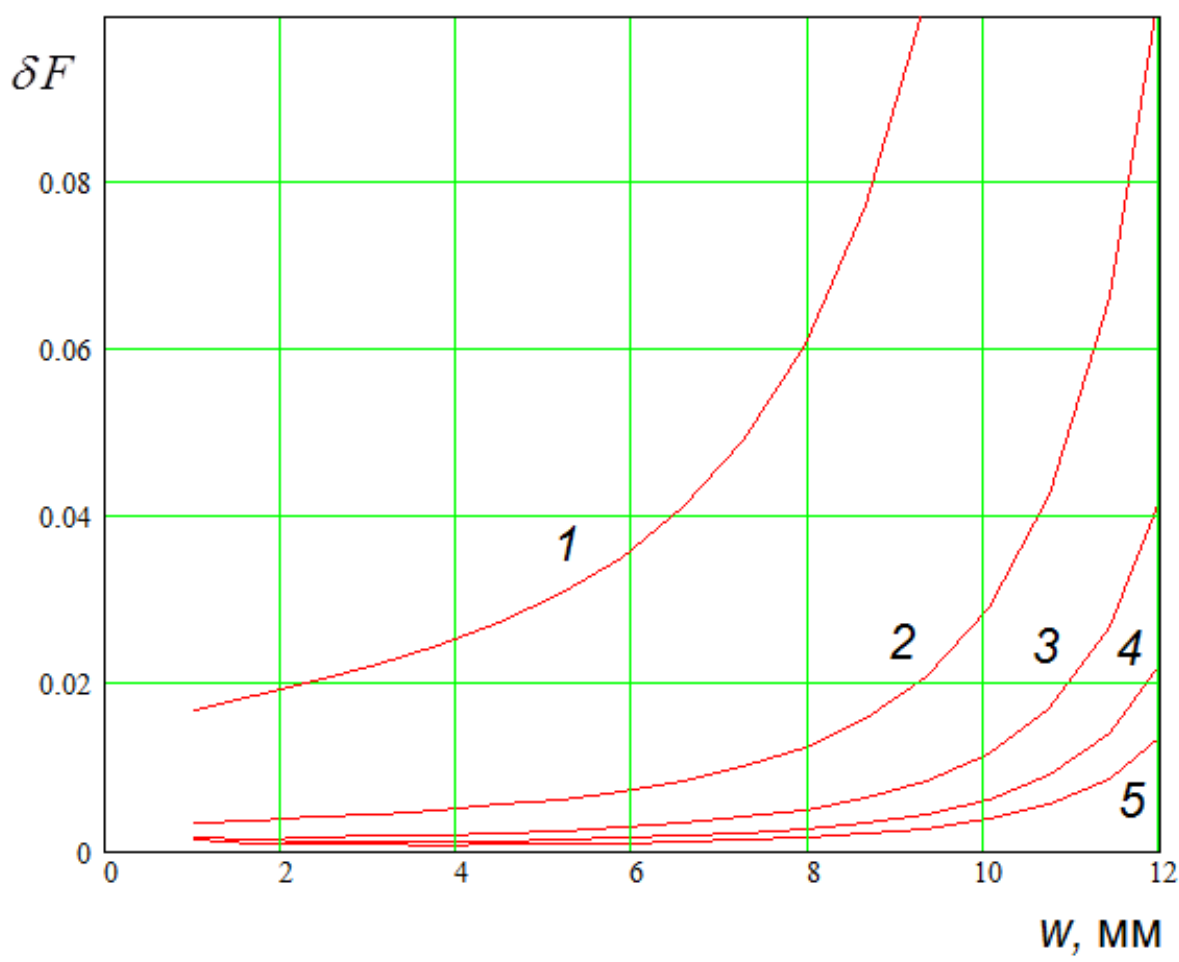

Fig. 6. Dependence of functional $\delta F$ on distance between conductors

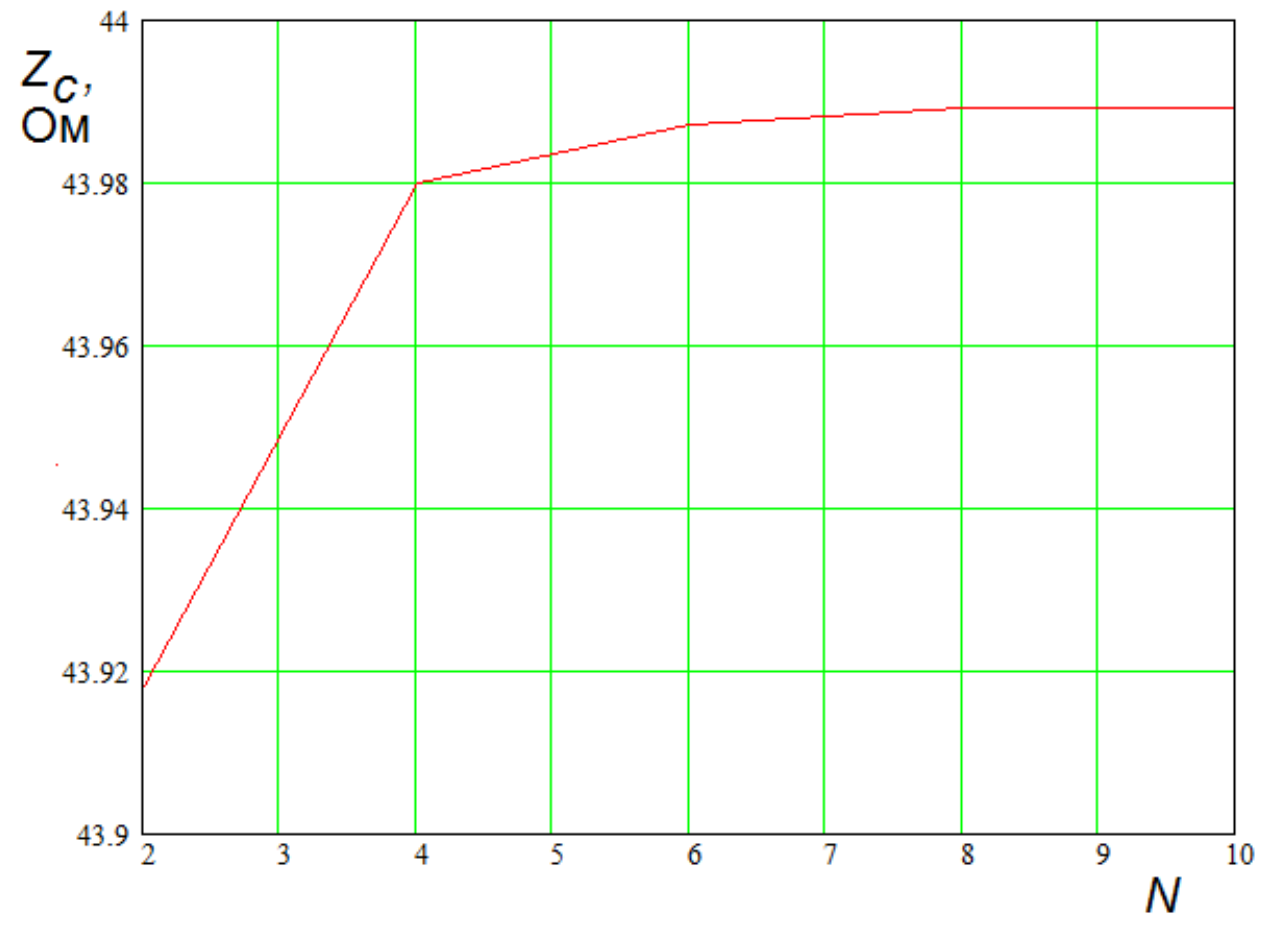




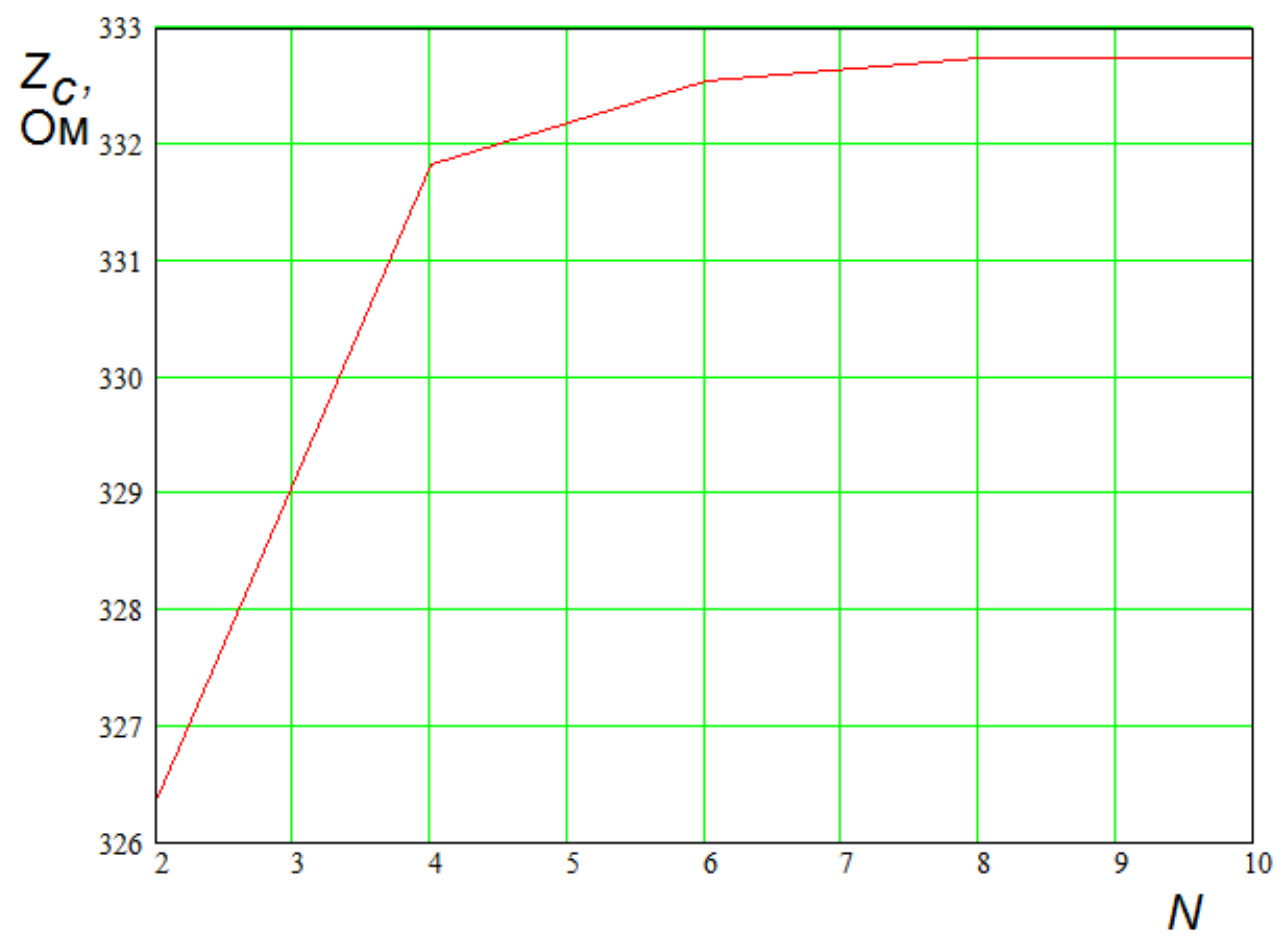

b

Fig. 7. Convergence of integral parameter $Z_{c}$

Different speed of convergence for integral and primary parameters is character for integral equation solution by Galerkin's method. It is a known fact [13] that integral parameters obtained by this method are stable relatively small variations of solution for field. As a result even sufficient errors in field have negligible influence on integral parameters. This property is an advantage of Galerkin's method that allows us to use a limited number of base functions for calculation of practically interesting parameter $Z_{c}$.

\section{Results of numerical solution of integral equation}

The analyzed structure is relatively simple because it has only one free parameter $q=w / P$. In addition because $T$ - wave field satisfies Laplas equation it does not depend on frequency. Dependence of characteristic impedance on parameter $q$ is shown in fig. 8 . The impedance varies from $q W_{0}$ when $q<<1$ to $W_{0}$ for $q=1$.

The following approximate expression is obtained for function $Z_{c}(q)$ : 


$$
Z_{c}(q)=q W_{0}\left(1+1.273 q^{2}(1-q)\right) .
$$

Formula (17) describes characteristic impedance with relative error less than $2 \%$ and it may be used for solution of engineering problems.

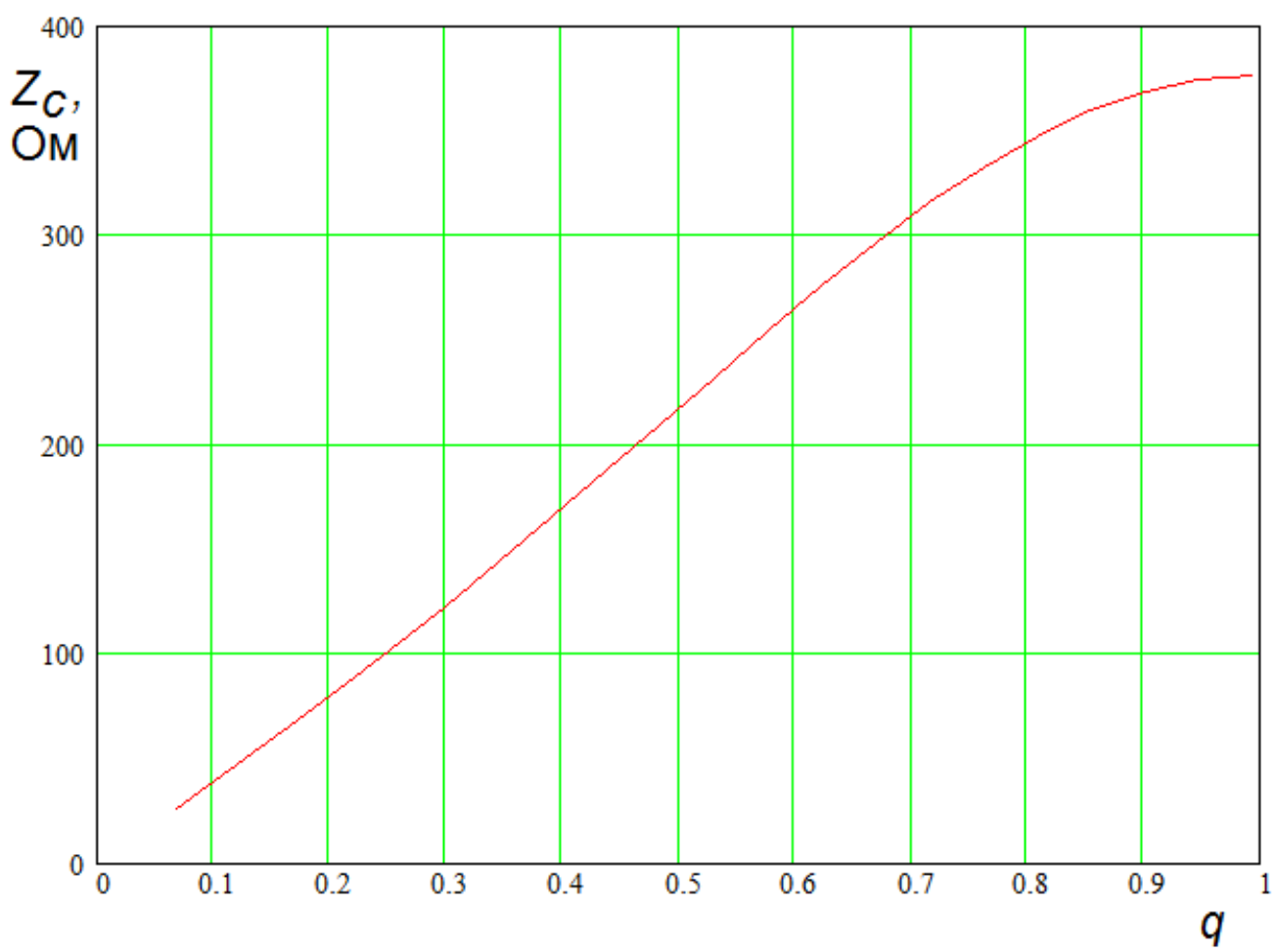

Fig. 8. Characteristic impedance versus parameter $q$

\section{Conclusion}

Thus in this work an approach for analysis of a dominant eigen mode of an infinite array of square metal rods is presented. Numerical algorithm based on this approach demonstrates high efficiency and it may be used for the second section synthesis of a dual section UWB array.

The work is performed in the frame of state assignment (project 0030-20190014) and is supported by Russian Foundation of Basic Research (project No. 1807-00655 a).

\section{References}

1. Schaubert D., Kasturi S., Elsallal M.W., Van Cappellen W. Wide bandwidth Vivaldi antenna arrays - some recent developments. Proc. EuCAP 2006. Nice. France. Nov. 2006. P. 1. 
2. Holter H., Chio T., Schaubert D.H. Elimination of impedance anomalies in single-and dual-polarized endfire tapered-slot phased arrays. IEEE Trans. AP. 2000. Vol.48. No.1. P. 122.

3. Song Y., Jiao Y.-C., Wang N.-B., Zhang T.-L., and Zhang F.-S. An 8-element Tapered Slot Antenna Array with a Bandwidth in Excess of 16.5:1. Proceedings of PIERS Symp. 2010. Xi'an, China, March 22-26, pp. 891-894.

4. Chan K.M., an. A.E.-C., Rambabu K. Decade Bandwidth Circularly Polarized Antenna Array. IEEE Trans. 2013. Vol. AP-61. No. 11. pp. 5435-5443.

5. Janaswamy R., Schaubert D. H. Analysis of the tapered slot antenna. IEEE Trans. 1987. Vol. AP-35. No. 9. pp. 1058- 1064.

6. Bankov S.E. Effects of blindness and depolarization in Vivaldi antenna arrays. Proc. Of the $4^{\text {th }}$ All Russian Microwave Conference. Moscow, November. 2016. pp. 265-269 (in Russian).

7. E. Acedo, E. Garcia, V. González-Posadas, J. Vázquez-Roy, R. Maaskan, D. Segovia. Study and design of a dierentially-fed tapered slot antenna array. IEEE Trans. AP. Vol. 58. No. 1. P. 68.

8. Bankov S.E. Theoretical study of a dual section ultra wideband antenna array. Journal of Communications Technology and Electronics. 2018. Vol. 63. No. 6. pp. 527-540.

9. Bankov S.E., Kurushin A.A., Gutzait E.M. Reshenie opticheskikh $i$ mikrovolnovykh zadach $s$ poomoshchyu HFSS [Solution of optical and microwave problems with the help of HFSS]. Moscow, Publishing house “Orcada". 2012. (In Russian)

10. Markov G.T., Chaplin A.T. Vozbuzhdenie elektromagnitnykh voln [Electromagnetic waves excitation]. Moscow, "Radio i Sviaz"” Publ., 1983. (In Russian)

11. Noble B. Wiener-Hopf technique. London-New York-Paris-Los Angeles, Pergamon Press. 1958.

12. Janke E., Hemde F., Loesh F. Tafeln hohererer funktionen. Stuttgart, B.G. Teubner Verlagsgeselschaft. 1960 (in German). 
13. Nikolsky V.V. Variatsionnyye metody dlya vnutrennikh zadach elektrodinamiki [Variational methods for internal electromagnetic problems]. Moscow, "Nauka” Publ., 1967. (In Russian)

\section{For citation:}

S. E. Bankov. Dominant T-mode of a cruciform waveguide Zhurnal Radioelektroniki - Journal of Radio Electronics. 2019. No. 9. Available at http://jre.cplire.ru/jre/sep19/6/text.pdf DOI 10.30898/1684-1719.2019.9.6 